\title{
SPACES IN WHICH COMPACT SETS HAVE COUNTABLE LOCAL BASES
}

\author{
RALPH R. SABELLA
}

\begin{abstract}
D_{1}$-spaces and coconvergent spaces are examples of spaces in which compact sets have countable local bases ( $D_{0}$-spaces). Among the results related to $D_{0}$-spaces given in this paper is a sufficient condition under which such spaces are coconvergent. In relation to a question posed by F. B. Jones, it is shown that a topological property sufficient for semimetrizable spaces to be developable is that they be coconvergent. Coconvergence implies metrizability in stratifiable spaces; it is shown in this paper that a $D_{0}$-space is metrizable if there exists a stratification satisfying a nesting-like condition.
\end{abstract}

An open neighborhood assignment (ONA) is a function

$$
U: X \times N \rightarrow \bigcup\{\Re(x): x \in X\}
$$

such that $x \in U(x, n) \equiv U_{n}(x)$, where $X$ is a topological space, $N$ is the set of natural numbers and $\eta(x)$ is the open neighborhood system of $x$. If $U$ is an ONA then the sequence $\left\{y_{n}\right\}$ is $U$-linked to $\left\{x_{n}\right\}$ if $y_{n} \in U_{n}\left(x_{n}\right)$ for all $n$. Using the notation $C p\left\{x_{n}\right\}$ for the set of cluster points of $\left\{x_{n}\right\}$, we define a space to be coconvergent (contraconvergent) if $C_{p}\left\{x_{n}\right\}$ $C C p\left\{y_{n}\right\}\left(C p\left\{x_{n}\right\} \supset C p\left\{y_{n}\right\}\right)$ whenever $\left\{y_{n}\right\}$ is $U$-linked to $\left\{x_{n}\right\}$. If on $X$ there is an ONA $U$ having some property $P$, we shall say " $X$ is $P$ " or " $U$ is $P . "$ If $x_{0}$ is a limit point of $\left\{x_{n}\right\}$, then $\Delta\left(x_{n}, x_{0}\right) \equiv\left\{x_{k}: k=0\right.$, $1,2, \ldots\}$.

Coconvergence can be characterized in terms of countably based compact sets. From [6] we have

Proposition 1. $X$ is coconvergent iff there is an ONA $U$ on $X$ such that for any compact set $K$ and open $R$ containing $K$ there is a $k \in N$ for which $\left\{\left\{U_{k}(x): x \in K\right\} \subset R\right.$.

Received by the editors October 10, 1973 and, in revised form, January 31, 1974. AMS (MOS) subject classifications (1970). Primary 54E35; Secondary 54D99.

Key words and phrases. Open neighborhood assignments, $U$-linked sequences, coconvergent, contraconvergent, stratifiable spaces, Nagata spaces. 
A $D_{1}$-space is defined by C. E. Aull [1] as a space in which every closed set $F$ has a countable local base $\left\{U_{n}(F)\right\}$, i.e. for each $n \in N$, $U_{n}(F)$ is an open set containing $F$, and if $F \subset R$, where $R$ is open, then $U_{k}(F) \subset R$ for some $k \in N$. Remaining with Aull's nomenclature we introduce

Definition 1. A space is $D_{0}$ if each compact set has a countable local base.

Without loss of generality we will always assume a given local base for a compact set to be nested. Trivially, every $D_{0}$-space is first countable; $T_{2}, D_{1}$-spaces, as well as coconvergent spaces, are $D_{0}$-spaces. Pointcountable [1] and, hence, second countable spaces are also $D_{0}$-spaces.

A first countable space is equivalent to one on which there is an ONA $U$ such that whenever $\left\{x_{n}\right\}$ is $U$-linked to the constant sequence $\{x\}$ then $\left\{x_{n}\right\}$ converges to $x$. The following is an analogue of this characterization:

Proposition 2. $X$ is a $D_{0}$-space iff for each compact $K \subset X$ there is a sequence $\left\{U_{k}(K)\right\}$ of open sets containing $K$ such that if $x_{n} \in U_{n}(K)$ for all $n$ then $\left\{x_{n}\right\}$ clusters in $K$.

Proof. Say $X$ is a $D_{0}$-space with $\left\{U_{k}(K)\right\}$ a local base for the compact set $K$. If for all $n, x_{n} \in U_{n}(K)$ but $\left\{x_{k}\right\}$ does not cluster in $K$, then there is an $m \in N$ such that for all $n>m, x_{n} \notin K$. Hence, $K \subset X-\operatorname{cl}\left\{x_{n}: n>m\right\}$, and for some $k>m, U_{k}(K) \subset X-\operatorname{cl}\left\{x_{n}: n>m\right\}$, which contradicts the condition that $x_{k} \in U_{k}(K)$.

Conversely, assume the condition to be satisfied. If $K$ is compact and contained in the open set $R$, and if $x_{n} \in U_{n}(K)-R$ for all $n$, then for some $x \in K, x \in C p\left\{x_{n}\right\}$. But $R \in \Re(x)$ and contains none of the $x_{n}$, which is a contradiction.

In coconvergent spaces the characterizing condition given in Proposition 1 is equivalent to the existence of an ONA $V$ such that for each $\Delta\left(x_{n}, x_{0}\right),\left\{\bigcup\left\{V_{n}(x): x \in \Delta\left(x_{k}, x_{0}\right)\right\}\right\}_{n=1}^{\infty}$ is a local base for $\Delta\left(x_{n}, x_{0}\right)$ [6]. As the next proposition and following example show, no such equivalence exists for $D_{0}$-spaces.

Proposition 3. Let $X$ be a $T_{1}$-space. Then $X$ is first countable iff each $\Delta\left(x_{n}, x_{0}\right)$ has a countable local base.

Proof. The sufficiency part is immediate. The converse follows by choosing a nested first countable ONA $U$ on $X$ and noting that, for any 
$\Delta\left(x_{n}, x_{0}\right)$ and each $k$, there is an $n_{k}$ such that $x_{n} \in U_{k}\left(x_{0}\right)$ for $n^{\prime}>n_{k}$. It follows that $\left\{B_{k, j}\left(\Delta\left(x_{n}, x_{0}\right)\right)\right\}_{k, j=1}^{\infty}$ is a local base for $\Delta\left(x_{n}, x_{0}\right)$ where

$$
B_{k, j}\left(\Delta\left(x_{n}, x_{0}\right)\right)=U_{k}\left(x_{0}\right) \cup\left[\bigcup_{r=1}^{n_{k}} U_{j}\left(x_{r}\right)\right] .
$$

The following is an example of a first countable, stratifiable space which is not a $D_{0}$-space:

Let $X=\left\{(x, y) \in R^{2}: y \geq 0\right\}$. Define $U$ by

$$
\begin{aligned}
& U_{n}(a, b)=\{(a, y) \in X:|y-b|<1 / n\} \text { if } b>0 . \\
& U_{n}(a, 0)=\{(x, y) \in X: y<1 / n, 0<|x-a|<1 / n\} \cup\{(a, 0)\} .
\end{aligned}
$$

$U$ defines a first countable, $T_{1}$ topology on $X$. The set $K=\{(x, 0): 0 \leq$ $x \leq 1\}$ is compact. Assume it has a local base $\left\{B_{n}(K)\right\}$ and let $L_{x}=$ $\{(x, y): y>0\}$. For each $n$ let $K_{n}=\left\{(x, 0) \in K: L_{x} \cap B_{n}(K)=\varnothing\right\}$. It follows that $K=\bigcup_{n=1} K_{n}$. By Baire's category theorem there are an integer $m$ and an interval $\{(x, 0): c \leq x \leq d\}$ in which $K_{m}$ is dense. There is a $k$ such that $U_{k}(c, 0) \subset B_{m}(K)$ and a $\bar{x} \neq c$ for which $(\bar{x}, 0) \epsilon$ $U_{k}(c, 0) \cap K_{m}$. Hence, $(\bar{x}, 1 / 2 k) \in U_{k}(c, 0) \subset B_{m}(K)$, i.e. $L_{\bar{x}} \cap B_{m}(K) \neq \varnothing$, contradicting $(\bar{x}, 0) \in K_{m}$. Hence $X$ is not a $D_{0}$-space.

A space is $D_{1}$ if it is perfectly normal and countably compact [1]. For $D_{0}$-spaces where we require the weaker condition that compact sets (rather than closed sets) have countable local bases, is perfect normality sufficient? The above example being stratifiable, and hence perfectly normal [2], provides us with an answer in the negative. However, we are able to weaken the countably compact condition to rim compactness, i.e. spaces in which for each $x \in X$ and $U \in \Re(x)$ there is a $V \in \Re(x)$ such that $V \subset U$ and bdry $V$ is compact.

Proposition 4. If $X$ is regular and rim compact, it is $D_{0}$ iff every compact set is a $G_{\delta}$.

Proof. Let $K$ be compact and $U_{n}(K)$ be a nested $G_{\delta}$ of $K$. If $R$ is open and contains $K$, there is a finite number of elements of $K, x_{1}, \cdots, x_{k}$ and open sets $V\left(x_{1}\right), \ldots, V\left(x_{k}\right)$ with $V\left(x_{i}\right) \in \eta\left(x_{i}\right)$ such that bdry $V\left(x_{i}\right)$ is compact and contained in $R$. Hence, $B=\operatorname{bdry}\left(\bigcup_{i=1}^{k} V\left(x_{i}\right)\right)$ is compact. 
The sequence $\left\{X-\operatorname{cl} U_{n}(K)\right\}$ covers $X-\bigcup_{i=1}^{k} V\left(x_{i}\right)$ and, hence, $B$. It follows that there is an $n \in N$ such that $B \subset X-\operatorname{cl} U_{n}(K)$ and, therefore, $U_{n}(K)$ $C R$.

The converse is true for any $D_{0}$-space and follows from Proposition 2.

Lemma 1. The following are equivalent:

(a) $X$ is coconvergent.

(b) $X$ is a $D_{0}$-space, each compact set $K$ having a local base $\left\{U_{n}(K)\right\}$ such that if $K_{1} \subset K_{2}$, both compact, then $U_{k}\left(K_{1}\right) \subset U_{k}\left(K_{2}\right)$ for all $k$.

(c) $X$ is a space in which each $\Delta\left(x_{n}, x_{0}\right)$ has a local base $\left\{U_{k}\left(\Delta\left(x_{n}, x_{0}\right)\right)\right\}$ such that $U_{k}(x) \subset U_{k}\left(\Delta\left(x_{n}, x_{0}\right)\right)$ for each $x \in \Delta\left(x_{n}, x_{0}\right)$ and all $k$.

Proof. Let $U$ be a nested coconvergent ONA on $X$. For each compact $K$ let $U_{n}(K)=\bigcup\left\{U_{n}(x): x \in K\right\}$. By Proposition $1,\left\{U_{n}(K)\right\}$ is a local base for $K$. It follows immediately that if $K_{1} \subset K_{2}$, both compact, then $U_{n}\left(K_{1}\right)$ $C U_{n}\left(K_{2}\right)$ for all $n$.

Since each $\Delta\left(x_{n}, x_{0}\right)$ is compact, (c) trivially follows from (b). As noted in the paragraph preceding Proposition 3, the condition that there exists an ONA $V$ on $X$ such that for any $\Delta\left(x_{n}, x_{0}\right)$, $\left\{\bigcup\left\{V_{k}(x): x \in \Delta\left(x_{n}, x_{0}\right)\right\}\right\}_{k=1}^{\infty}$ is a local base for $\Delta\left(x_{n}, x_{0}\right)$ is equivalent to the existence of a coconvergent ONA on $X$. It easily follows from this that (c) implies (a).

Lemma 2. Let $X$ be a $T_{1}$-space. Then $X$ is developable if it is coconvergent and semistratifiable.

Proof. Since $X$ is semistratifiable, there is an ONA $V$ such that if the constant sequence $\{x\}$ is $V$-linked to $\left\{x_{n}\right\}$, then $\left\{x_{n}\right\}$ converges to $x$ [4]. Without loss of gene rality we may choose an ONA $U$ which is semistratifiable and coconvergent. For each $n$ let $\mathcal{S}_{n}=\left\{U_{n}(x): x \in X\right\}$. The sequence $\mathscr{O}_{n}$ is a development $\mathrm{f}$ or $X$, for if there is an $x \in X$ and $R \epsilon$ $\Re(x)$ such that for all $n$ there are sequences $\left\{x_{n}\right\}$ and $\left\{y_{n}\right\}$ for which $\{x\}$ is $U$-linked to $\left\{x_{n}\right\}$ and $y_{n} \in U_{n}\left(x_{n}\right)-R$, it would follow that $x \in C p\left\{y_{n}\right\}$, which contradicts the way in which $\left\{y_{n}\right\}$ was chosen.

R. W. Heath in [5] gives an example of a semimetric (hence semistratifiable), $D_{0}$-space which is not developable. The example was in answer to a conjecture by F. B. Jones, viz. a topological property sufficient for a semimetrizable space to be a developable space is that it be $D_{0}$. Lemma 2 
shows that coconvergence is one topological property which will work. It is is not known whether, in fact, coconvergence is not also a necessary condition.

Proposition 5. $X$ is a Moore space if it is regular, rim compact and semistratifiable.

Proof. Let $U$ be a semistratifiable ONA on $X$. Letting $U_{n}(K) \equiv$ $\mathcal{U}\left\{U_{n}(x): x \in K\right\}$ for any compact set $K$, it follows from Proposition 2 that $\left\{U_{k}(K)\right\}$ is a $G_{\delta}$, and using the proof of Proposition 4, it is a local base for $K$. Now by Lemma $7, X$ is coconvergent, and by Lemma 2, it is developable.

Proposition 6. If $X$ is locally compact, $T_{2}$ and its one point compactification is perfectly normal, then $X$ is a $D_{0}$-space.

Proof. By Proposition 4, $X^{*}$, the one point compactification of $X$, is a $D_{0}$-space. If $K$ is compact in $X$, it is compact also in $X^{*}$ and has a local base $\left\{U_{n}(K)\right\}$. But then $\left\{U_{n}(K) \cap X\right\}$ is a local base for $K$ in $X$.

The last two propositions are applications of $D_{0}$-spaces to stratifiable spaces. From [7] we have

Proposition 7. $X$ is metrizable iff it is stratifiable and coconvergent.

We have from the example following Proposition 3: not every stratifiable space is $D_{0}$. Can the conditions of Proposition 7 be weakened, viz. is it sufficient that a stratifiable space be $D_{0}$ to be metrizable? We have only a partial answer in

Proposition 8. $X$ is metrizable if it is stratifiable and $D_{0}$ with each compact set $K$ having a local base $\{X-(X-K)-\}$. (We are using $\left\{R_{n}\right\}$ to denote an increasing stratification of the open set $R$.

Proof. If $K_{1}$ and $K_{2}$ are compact with $K_{1} \subset K_{2}$, then $\left(X-K_{2}\right)_{n} \subset$ $\left(X-K_{1}\right)_{n}$ for all $n$ and, hence, $U_{n}\left(K_{1}\right) \subset U_{n}\left(K_{2}\right)$, which by Lemma 1 and Proposition 7 give metrizability.

Proposition 9. If $X$ is stratifiable and rim compact, it is metrizable.

Proof. By Proposition 5, $X$ is a Moore space. But stratifiability implies paracompactness which is sufficient in Moore spaces for metrizability. 


\section{REFERENCES}

1. C. E. Aull, Closed set countability axioms, Nederl. Akad. Wetensch. Proc. Ser. A 69 = Indag. Math. 28 (1966), 311-316. MR 33 \#7973.

2. C. J. R. Borges, On stratifiable spaces, Pacific J. Math. 17 (1966), 1-16. MR $32 \# 6409$.

3. J. G. Ceder, Some generalizations of metric spaces, Pacific J. Math. 11 (1961), 105-125. MR 24 \#A 1707.

4. G. D. Creede, Concerning semi-stratifiable spaces, Pacific J. Math. 32 (1970), 47-54. MR 40 \#8006.

5. R. W. Heath, On certain first-countable spaces, Ann. of Math. Studies, no. 60, Princeton Univ. Press, Princeton, N.J., 1965, pp. 103-113.

6. R. R. Sabella, Convergence properties of neighboring sequences, Proc. Amer. Math. Soc. 38 (1973), 405-409.

7. - Properties of neighboring sequences in stratifiable spaces, Proc. Amer. Math. Soc. (submitted).

DEPARTMENT OF MATHEMATICS, CALIFORNIA STATE UNIVERSITY, NORTHRIDGE, CALIFORNIA 91324 